\title{
THE SALZBURG MEETING OF THE DEUTSCHE MATHEMATIKER VEREINIGUNG.*
}

The twentieth annual meeting of the Deutsche Mathematiker-Vereinigung was held at Salzburg, Austria, September 19 $-25,1909$, in affiliation with the eighty-first convention of the Society of German naturalists and physicians.

An informal reception was held in the Kurhaus on the evening of September 19, and the first general session of all the sections and affiliated societies took place in the Aula the following morning.

The five sessions of the Vereinigung were held at the Realschule. The following papers were read :

(1) F. EngeL, Greifswald, "Hermann Grassmann."

(2) E. WAELSCH, Brünn, "Applications of the theory of invariants of binary forms."

(3) G. Prck, Prague, "Differential equations of the periods of hyperelliptic functions."

(4) Miss E. Noether, Erlangen, "On the theory of invariants of forms of $n$ variables."

(5) G. KoHs, Vienna, "On a group of theorems of projective geometry."

(6) R. Rothe, Clausthal, "On the theory of isothermal surfaces."

(7) R. Müller, Darmstadt, "On the instantaneous motion of similarly varying plane systems."

(8) R. Menmke, Stuttgart, "Contributions to the kinematics of rigid spatial systems, and those varying in affinity."

(9) M. GRÜBLER, Dresden, "The criterion for constrained motion of a system of screws."

(10) E. Salkowski, Charlottenburg, "On a remarkable class of deformation surfaces of hyperboloids of revolution."

(11) H. Wiener, Darmstadt, "Application of kinematic considerations to the construction of curves."

(12) E. STÜBler, Stuttgart, "The systems of accelerations in the motion of a rigid body."

(13) H. Wiener, Darmstadt, " On some new kinematic and geometric models."

\footnotetext{
* Translated from Dr. Dintzl's manuscript by Professor Virgil SNyder.
} 
(14) R. Skutsch, Dortmund, "Explanation of some demonstration apparatus."

(15) H. Jung, Hamburg, " Report on the theory of algebraic functions of two variables."

(16) S. Günther, Munich, "Mathematical and physical geography of Leonhard Euler."

(17) E. Hoppe, Hamburg, "The sexagesimal system and the division of the circle of the Babylonians."

(18) L. G. Du PASquier, Zürich, "Concerning the question of the most suitable base for a system of numbers."

(19) W. Velton, Kreuznach, "The development of elliptic functions."

(20) H. Hahn, Vienna, "Report on the theory of linear integral equations."

(21) A. KonN, Munich, "Solution of the linear integral equation by the method of successive approximations."

(22) W. Wirtinger, Vienna, "Concerning conformal representation by means of abelian integrals."

(23) O. Perron, Munich, "On the behavior of the integrals of linear difference equations at infinity."

(24) L. G. DU PASquier, Zürich, "On integral tettarions."

(25) E. Timerding, Strassburg, "Kinematic models."

(26) R. Menmke, Stuttgart, "The calculating machine 'Euclid.",

(27) E. Papperitz, Freiberg, "The kinodiaphragmatic projection, a new method of geometric representation."

(28) F. ENGEL, Greifswald, "On a family of curves invariant to a given differential expression."

(29) E. MüLLER, Vienna, "Suggestions for shaping a course in descriptive geometry in technical schools and universities."

(30) E. Czuber, Vienna, "The measure of mortality."

With the exception of numbers 3 and 6 , abstracts of the papers are given below, the numbers corresponding to those in the list above.

1. Professor Engel gave a brief sketch of the mathematical development of Grassmann, which is all the more remarkable since he studied under no one. It is probably for this reason that his writings have not found more recognition, and have not exerted the influence on mathematical development that the importance and novelty of the ideas contained in them deserve.

2. Professor Waelsch generalized his definition of a spherical 
harmonic in three-dimensional space, as the complete transvection of a binary form $\alpha_{\xi}^{2 n}$ on the $n$th power of the quadratic form $(x+i y) \xi_{1}^{2}-2 i z \xi_{1} \xi_{2}+(x-i y) \xi_{2}^{2}$, to apply to four-dimensional space. The harmonic of $R_{4}$ appears as the bilateral complete transvection of the bilinear form $a_{\xi}^{n} b_{\eta}^{n}$ on the $n$th power of the form

$$
(x+i y) \xi_{1} \eta_{1}+(u-i z) \xi_{1} \eta_{2}-(u+i z) \xi_{2} \eta_{1}+(x-i y) \xi_{2} \eta_{2} .
$$

The methods of binary invariants are not applicable to the euclidean geometry of higher spaces. The theory can therefore be regarded as a specific auxiliary for euclidean geometry of three and of four dimensions.

4. The theorems relating to the composition of forms in a ternary field are known. Their generalization to forms of $n$ variables was impossible because no comprehensive symbolism for such forms existed. Miss Noether has developed such a symbolism by means of products of matrices. The two fundamental theorems, that concerning invariant processes and that regarding the finiteness of the system of forms, readily appear by this method.

5. The fundamental concept of Professor Kohn's paper is the "Wurf" on $n$ elements in $R_{d}(n>d+2)$. These Würfe are arranged in pairs ; for every one of $n$ elements in $R_{d}$ an associated Wurf of $n$ elements in $R_{n-d-2}$ is determined. By this means a large number of isolated theorems in projective geometry are brought into systematic relation, including Clifford's anharmonics, Rosanes's linearly independent systems of pairs of points, and Sturm's problem of projectivity.

7. Professor Müller considered first the motion through three consecutive phases, determined the center of curvature of the path curve of an arbitrary point of the system, the $(1,2)$ correspondence between the systems of moved points and the associated centers of curvature, and the curvature of the envelope; second, the corresponding motion through four or more such positions, introducing stationary curvature and Burmester points, as well as the locus of the pole. Finally, the determination of the phases by means of the instantaneous positions of three arbitrary points, and the introduction of branch phases.

8. Professor Mehmke discussed the torsion of an element $x$ 
of an orbit, under the most general conditions. A relation be tween the bisecant of the inflexional curve through $x$, the torsion of $x$ and its position on the bisecant on the one hand, and the angle of contingence and the binormal component of the hyperacceleration on the other was found, which are analogous to the formula for the normal acceleration of a plane system. The formula was generalized for certain hyperspaces, and interpretations given for the behavior at singular points.

9. The condition under which a closed system of screws consisting of $n$ members and $s$ screws may still have constrained motion was given by Professor Grübler as $5 s-6 n+7=0$, when the axes of the screws are independent. For systems of rotation the criterion is the same. If the axes of revolution intersect, giving rise to spherical motion, the criterion reduces to that of plane systems, $2 s-3 n+4=0$. This last relation is also true for translation.

10. The surfaces here considered appear in connection with the Bertrand curves in the following way : The rectifying cuspidal edges of two associated Bertrand curves have the same rectifying surface. After proving this theorem Professor Salkowski considered various special cases. The curves which give rise to cylinders are obtained by regarding a plane as the developable of an asteroid. The latter should now be twisted, keeping the curvature invariant, into a, helix. The axes of the curve will become Bertrand curves and the altitudes of the triangles formed by the axes and a tangent will define two deformation surfaces of a hyperboloid of revolution.

11. When a point describes a plane curve the curvature is determined by the velocity and acceleration. Professor Wiener showed by means of numerous examples how to construct the tangent and radius of curvature, when the law of motion is properly chosen. If the circle of osculation be replaced by the parabola of osculation, these constructions can be simplified, and generalized to properties of contact of any order.

12. Professor Stübler investigated the instantaneous motion of a rigid system defined by the vector of the velocity of translation $v$, the rotation vector $n$, and the position of the axis of the screw. He showed how to construct the vector defining the acceleration, or conversely, when this is given, to find the 
preceding elements. Then followed a construction for the axis of curvature, and the equation for the curve of inflexions, obtained as the locus of the centers of all possible accelerations compatible with the system. The case in which the velocity of rotation is perpendicular to the vector was studied in detail.

13. Professor Wiener exhibited two groups of models, invented by the author, eight to illustrate linkage quadrilaterals, and thirteen to illustrate linkage surfaces. The necessary and sufficient condition that a ruled surface be such a surface with regard to its generators and a system of directrix curves was given. A planigraph constructed on these principles was capable of drawing a complete circle of $64 \mathrm{~cm}$. with an apparatus $40 \mathrm{~cm}$. high.

14. Dr. Skutsch exhibited an apparatus for demonstrating the theorem of Möbius regarding the equilibrium of varying systems, keeping similarity as an invariant property.

15. In the report of Professor Jung, the investigation was restricted to linear systems of curves and the various invariant numbers that appear in functions of two independent variables.

16. It was shown by Professor Günther that the productions of Euler in mathematical geography were extensive and important, including the determination of latitude and longitude, map drawing, and the shifting of the earth's axis.

17. Professor Hoppe showed that the sexagesimal system arose from the efforts of the Babylonians to determine and define directions. The fundamental angle was not a right angle, but that of the equilateral triangle. This was divided into ten equal parts, and later into 60 equal parts. The theory is confirmed by numerous writings in the cuneiform characters.

18. Dr. du Pasquier arrived at the conclusion that the most suitable basis number is 4 .

19. Starting from the differential equation defining the logarithm of an elliptic function, Dr. Velten derived the expansion in series of the Jacobi function.

20. On account of the limited time available for preparation Dr. Hahn confined his report to the Fredholm equation. He considered the solution by means of the Neumann series, the 
derivation of the equation from an algebraic one by a limiting process, the memoirs of Plemelj and Goursat on the unsymmetric kernel, those of Hilbert and Schmidt on the symmetric kernel, and finally the method of Hilbert of an infinite number of variables.

21. The paper of Dr. Korn is an extension of that in the Comptes Rendus of June 24, 1907, by means of the methods of Poincaré, published in the Palermo Rendiconti in 1904, analogous to that employed in the solution of the differential equation $\Delta \phi+\kappa^{2} \phi=f$. Results already found by Hilbert, Fredholm, and others, were rediscovered, and an explanation of an infinite kernel of degree $\lambda<1$ was given.

22. Professor Wirtinger first showed that every Riemann surface of an abelian integral can be uniquely obtained by the repetition of a finite number of convex rectilinear polygons: The process was carried through for normal elliptic integrals of the second kind, and for hyperelliptic integrals of the first kind. In the second case the dissection of the surface may not be unique, so that each sheet may be mapped upon a plane hexagon having given properties. From this standpoint it follows immediately that the transformation group of the periods can for $p=2$ be generated by two operators.

23. When in the difference equation of order $r$

$$
\begin{aligned}
D_{\nu+r}+a_{1}^{(\nu)} D_{\nu+r-1}+a_{2}^{(\nu)} D_{\nu+r-2}+\cdots+a_{r}^{(\nu)} D_{\nu}=0 & \\
\left(a_{r}^{(\nu)}\right. & \neq 0) \quad(\nu=0,1,2, \cdots)
\end{aligned}
$$

the coefficients $a_{i}^{(\nu)}$ approach a power of $\nu$, say $a_{i}^{(\nu)} \doteq \nu^{\kappa_{i}}$, then the integrals approach $\nu$ !. The exponents of $\nu$ ! are functions of $\kappa$ only, and can be immediately found by a simple geometric construction. Dr. Perron discussed equations which are related to the above form, or can be generalized from it, and applied his results to linear differential equations.

24. The tettarions discussed by Dr. du Pasquier are defined as a special class of complex numbers that are related to linear substitutions. The idea of the field was developed and some fundamental theorems established regarding tettarion integers.

25. Professor Timerding exhibited his model, recently manufactured by Schilling, to illustrate systems varying in affinity. 
In them the Nurnberger rails are applied to construct triangles and tetrahedra.

26. Professor Mehmke mentioned, as two principal advantages of the calculating machine in question, the construction of the gear and the automatic division.

27. The procedure explained by Professor Papperitz and illustrated by the lantern consists essentially in the varying role of rapidly moving models which serve to generate a picture, to translate it, and to develop it. For example, consider two diaphragms with different width of slit, in rapid rotation. The intersections of the strips of light will generate a variety of curves. On the other hand, by means of a fixed slit, a model can be projected which will give rise to as great a variety of space curves on the changing surface of the model. Curves can also be generated by appropriate shadows.

28. The calculus of variations associates with every differential expression $w\left(x, y, y^{\prime}\right) d x$ a covariant system of curves, the extremals, for which the first variation of the integral $\int w d x$ vanishes. Professor Engel points out that the extremals are only the first links of an endless chain of systems of curves of this kind. If $\infty^{2}$ curves $c$ be defined by $y^{\prime \prime}=\phi\left(x, y, y^{\prime}\right)$ and $\int w d x$ defines the length of arc along a curve $c$, and if two particular curves of the system are fixed by having given tangents at a given point, and finally if $d s_{0}$ is the difference of their length of arc at the point of intersection, then a relation $\psi\left(x_{0}, y_{0}, y_{0}^{\prime}, d x_{0}, d y_{0}, d x_{0}^{\prime}, d y_{0}^{\prime}, d s_{0}\right)=0$ exists. If this relation be linear and homogeneous in the differentials, it is an extremal. If it be homogeneous and of degree $n$, a category of covariant curves results, and indeed the given $\phi$ defined by a partial differential equation. The concept can be generalized in various directions.

29. Professor Müller emphasized that descriptive geometry should be taught in the technical schools from the standpoint of its practical applications, and that elementary theoretical instruction in it should be provided in all secondary schools. The speaker then explained his own method, and illustrated it with over 300 drawings. He further urged that all candidates for positions as teachers should not only hear courses in descrip- 
tive geometry, but also take part in extensive constructive exercises.

30. Professor Czuber first pointed out that from the result of tl e investigations of the statistical reports furnished by 60 English and by 28 Austrian insurance companies it is clear that the probability of death is not only a function of the age, but also of the length of the term of insurance. Moreover, other factors must be considered, such as sex, occupation, and personal equations in medical certificates. The problem of the measure of mortality is not so much to determine the functional relation between these numbers, which now seems impossible, as to determine individual cases empirically. The details of this procedure were then illustrated by some numerical examples.

In the general sessions, mention should be made of the paper by Professor Einstein, Bern, "The recent changes which our views of the nature of light have undergone."

In the business meeting of the Vereinigung, which was held on Thursday, September 23, reports of the various committees and officers were read, and appointments for the following year were made. Professors Krause and Schoenflies retired from the executive committee, and their places were filled by the election of Professors E. Czuber and R. Müller. The library and bibliography committee was continued. Professor Rudio reported on the status of the publication of the works of Euler.

Ample provisions were made for social intercourse, two or more entertainments being held every evening. All the participants felt that it was a most successful occasion. The next meeting will be held in Königsberg.

E. DintzL.

\section{GERGONNE'S PILE PROBLEM.}

BY. DR. H. ONNEN, SR.

In volume I (1895), page 184, of the Bulletin, Professor L. E. Dickson has treated Dr. C. T. Hudson's solution of the problem :* To deal a pack of $a b$ cards into $a$ piles of $b$ cards each and so stack the piles after each deal that after the $n$th deal any selected card may be the $r$ th in the whole pack.

* Educational Times Reprints, 1868, vol. 9, pp. 89-91. I have tried in vain to lay my hand on Dr. Hudson's article. 